\title{
The Role of Global Longitudinal Strain in Early Detection of Doxorubicin Induced Cardiotoxicity in Breast Cancer Patients
}

\author{
Wisam Abbas Hasan ${ }^{1}$, Shokry faaz Al-saad ${ }^{1}$, Hassan Salim Aljumaily ${ }^{1}$ \\ ${ }^{1}$ College of medicine, University of Babylon, Iraq.
}

\begin{abstract}
Objective : Cardiotoxicity is a recognized side effect of many antineoplastic drugs, particularly anthracyclines which used for breast cancer treatment. The identification of patients at high risk for developing cardiotoxicity would be a good strategy to decrease mortality. the aims was to assess the incidence of chemotherapy induce cardiotoxicity and identifying the reduction in global longitudinal strain in various treatment cycles for the early detection of cardiotoxicity, prior to ejection fraction reduction.

Method: A prospective cohort study of confirmed and newly diagnosed breast cancer patients, who attend the Babylon oncology center in Marjan medical city between the fifth of January and the first of July of 2019.

Results: The mean patient's age was 51.3( \pm 10.5$)$ years, There is no association difference in systolic function during the follow up period. The reduction in global longitudinal strain by $8 \%-15 \%$ from baseline was found in 8 patients, while reduction $\geq 15 \%$ from baseline was found in only 2 patients during the follow up period.

Conclusions: Global longitudinal strain drop was observed three weeks after chemotherapy onward without significant reduction in ejection fraction.
\end{abstract}

Keywords: breast cancer; Doxorubicin; longitudinal strain

\section{Introduction}

Breast cancer is the high frequent cancer among women, effecting 2.1 million women yearly, it also causes the highest number of cancer-related deaths among women ${ }^{(1)}$. As result of that the breast cancer is presently a important public health and economical problem and many research on new therapies and monitoring their safety use, should be a priority ${ }^{(2)}$. Cancer therapy has studied in a recent years and it has been proved to risen average of cure in breast cancer, as well as to decrease the recurrences.

However the cardiac toxicity from antineoplastic therapy has become a notable risk of morbidity and mortality in cancer pateints. In patients who develop heart failure from chemotherapy, the mortality rate is increase to $60 \%$ by 2 years (3). Cardiotoxicity may appear early or late in the course of the disease, and have different clinical feature from subclinical myocardial dysfunction to irreversible heart failure or even death ${ }^{(4)}$. The cardiotoxicity refers to a direct influence of the chemotherapy on the entire CVS and also to an indirect effect by a thrombogenic status or to a hemodynamic flow alteration ${ }^{(5)}$. In 2016, the European Society of Cardiology has published a position paper recommending the diagnosis of cardiotoxicity occur when the ejection fraction $(\mathrm{EF})$ reduction $>10 \%$ for values below normality $(53 \%)^{(6)}$.

Early detection of cardiotoxicity has frequently depended upon many cardiac imaging to identify a reduction in LV function without signs or symptoms of heart failure (stage B heart failare $)^{(7)}$. The use of ejection fraction has important limitations.

The measurement of left ventricular ejection fraction is subject to technique-related variability, which can be higher than the thresholds used to define cardiotoxicity ${ }^{(8)}$.

The decreasing in left ventricular ejection fraction is often a late phenomenon, with failure to recover systolic function in up to $58 \%$ of patients despite intervention ${ }^{(9}$, 10). 
The common measurements allow only the late diagnosis of cardiac dysfunction, which might be mostly irreversible. Therefore, there is major need for other precise and reproducible parameters, able to diagnose the early, subclinical, LV dysfunction and can detect the patients at risk for rapid progression toward irreversible cardiac failure and who can benefit from early therapeutic treatment ${ }^{(11)}$. The using of tissue Doppler-based strain imaging and GLS has most constantly determined early myocardial changes during therapy, whereas STE in form of GLS appears to be the best measure ${ }^{(12)}$.

Global longitudinal strain (GLS) is used in clinical practice aimed at the early detection of changes in myocardial contractile function However, neither GLS use nor its cutoff value determined to predict cardiotoxicity have been standardized ${ }^{(13)}$.

The American Society of Echocardiography and the European Association of Cardiovascular Imaging have pointed that deformity changes prior ventricular dysfunction. A reduction $>15 \%$ in GLS, immediately after or during anthracycline treatment, was the most informative parameter to predict cardiotoxicity, while a reduction $<8 \%$ might exclude its diagnosis. However, there is a grey zone between those two values ${ }^{(14)}$.

Doxorubicin is an anthracycline widely used in many cytostatic treatment regimens, but has limited applicability due to cardiotoxic effect. This cardiotoxicity increases patient morbidity and mortality. The mechanisms of cardiotoxicity induced by anthracyclines include the formation of oxygen free radicals, destruction directly to the DNA, apoptosis, activation of immunological reactions in the myocardium, changes in calcium ions transport, histamine release, and activation of coagulation with the formation of thrombus in coronary arteries ${ }^{(15)}$.Doxorubicin-induced cardiotoxicity may be augmented by increased cumulative dose, association with other cytotoxic drugs, and patients' advanced age (16). In spite of this toxicity, anthracyclines stay the cornerstone of treatment in a lot type of malignancies, including lymphomas, leukemias, and sarcomas, and are still widely used in both advanced and early-stage breast cancer ${ }^{(17)}$. This study was palliated the early global longitudinal strain changes in breast cancer patients.

\section{Patients and Method}

This is a prospective cohort study included a convenient patients conducted in the fifth of January and the first of July of 2019 and who came to Babylon oncology center were asked to participate in this study. Sample size of 40 patients was included. The patients with Age $\geq 18$ years, newly diagnosis of breast cancer with neither antineoplastic treatment nor radiotherapy, Normal baseline echocardiography study and Antineoplastic treatment planning with doxorubicin. Exclusion patients with Lacking of accurately assessing GLS because of an inappropriate acoustic window, cardiac arrhythmias and/or non-sinus rhythms, Usage of beta-blockers and/or angiotensin-convertingenzyme inhibitors and/or angiotensin receptor blockers, Moderate or severe heart valve disease or Ischemic heart disease and Prior history of treatment with cardio toxic drug and thoracic radiotherapy

The baseline information was recorded in form of patient's age, medical history of DM, HT and dyslipidemia, smoking history, oncological history (side and type of cancer), weight and height was measured by researcher.

Heart rate was measured manually and blood pressure was measured by sphygmomanometer then patient underwent Doppler echocardiogram at the baseline, before initiating the doxorubicin $(60 \mathrm{mg} / \mathrm{m} 2$ IV infusion), three weeks after the first dose and three weeks after the second dose ( according to the protocol of Breast cancer chemotherapy). Data was analyzed using SPSS version 23 and used ANOVA test used to find association between variables. Echocardiographic examination was performed with the patient at rest in the left lateral position, using the Vivid E9-GE device (GE, Vingmed Ultrassound Horten, Norway), LCD 17" monitor, with image acquisition with a M5Sc transducer and harmonic imaging. All tests were performed with the same device. Sector and depth were adjusted to optimize the image. The measurements and image acquisition followed the recommendations of the American Society of Echocardiography and the European Association of Cardiovascular Imaging

The following echocardiographic variables were assessed: EF, calculated by using of the biplane Simpson's method, considering the normal value of EF $>54 \%$ for the female sex ${ }^{(18)}$.

According to the current recommendations ; diastolic function, evaluated by use of mitral flow with anterograde values of $\mathrm{E}$ wave and $\mathrm{A}$ wave, tissue Doppler of lateral mitral annulus, measures of S' wave (systolic velocity of the mitral ring) and ; indexed left 
atrial volume $\left(\mathrm{mL} / \mathrm{m}^{2}\right)$; tricuspid annular plane systolic excursion (TAPSE).

The GLS was acquired by use of Automated Functional Imaging (AFI) of three clips with images of the left ventricle on three apical views, so that all myocardial segments could be well visualized: 4-chamber, 2-chamber and 3-chamber views. The events of aortic valve opening and closure were marked. The images were acquired at a frame rate of $40-90 \mathrm{fps}(>$ $70 \%$ of heart rate).

\section{Results}

40 patients was included. No loss to follow up, the baseline information was showed in table -1-.

Table -1- Patients baseline characteristics.

\begin{tabular}{|l|l|l|l|}
\hline Variable & Mean $( \pm$ SD $)$ & Maximum & Minimum \\
\hline Age in years & $51.3( \pm 10.5)$ & 76 & 32 \\
\hline BMI in $\mathrm{Kg} / \mathrm{m} 2$ & $20.5( \pm 4.1)$ & 33.6 & 11.9 \\
\hline BSA in m2 & $1.7( \pm 0.1)$ & 1.9 & 1.4 \\
\hline HR in b/m & $78( \pm 7.3)$ & 95 & 64 \\
\hline SBP in mm Hg & $13.4( \pm 0.7)$ & 140 & 120 \\
\hline DBP in $\mathrm{mm} \mathrm{Hg}$ & $83.1( \pm 7.3)$ & 95 & 60 \\
\hline
\end{tabular}

History of diabetic was found in $12.5 \%$ of patients, $10 \%$ had a history of Dyslipidemia, $70 \%$ of patients had left breast cancer. Most of patients had invasive ductal type of breast cancer (82.5\%), table -2-.

Table -2- Patient medical history.

\begin{tabular}{|l|l|l|l|}
\hline \multicolumn{2}{|l|}{ Variable } & NO. & Percentage \\
\hline \multirow{3}{*}{ History of DM } & Yes & 5 & $12.5 \%$ \\
\cline { 2 - 4 } & No & 35 & $87.5 \%$ \\
\hline \multirow{3}{*}{ History of Dyslipidemia } & Yes & 4 & $10 \%$ \\
\cline { 2 - 5 } & No & 36 & $90 \%$ \\
\hline \multirow{3}{*}{ Laterality } & Right & 12 & $30 \%$ \\
\hline \multirow{3}{*}{ Type of cancer } & Left & 28 & $70 \%$ \\
\hline & Invasive ductal & 33 & $82.5 \%$ \\
\cline { 2 - 5 } & Lobular & 5 & 12.5 \\
\cline { 2 - 5 } & Others & 2 & $5 \%$ \\
\hline
\end{tabular}

The baseline ejection fraction reading was $66.2 \%( \pm 5.9)$ and there was no significant difference in mean between the three readings ( $\mathrm{p}$ value $>0.05$ ). Also for LAVI, TDI, TAPSE and GLS there were no significant difference in mean between the three readings ( $\mathrm{p}$ value $>0.05$ ), table $-3-$. 
Table -3- Comparison of echo parameters changes during follow up period.

\begin{tabular}{|c|c|c|c|c|}
\hline \multirow[b]{2}{*}{ Variable } & \multicolumn{3}{|l|}{ Mean \pm SD } & \multirow[b]{2}{*}{$P$ value } \\
\hline & Reading at base line & $\begin{array}{l}\text { Reading after } 3 \text { week } \\
\text { from first dose }\end{array}$ & $\begin{array}{l}\text { Reading after } 3 \text { week } \\
\text { from second dose }\end{array}$ & \\
\hline $\mathrm{EF} \%$ & $66.2 \%( \pm 5.9)$ & $66.8 \%( \pm 5.5)$ & $67.5 \%( \pm 5)$ & 0.58 \\
\hline LAVI & $26( \pm 3.8)$ & $26.7( \pm 3.9)$ & $26.4( \pm 3.9)$ & 0.72 \\
\hline TDI(s-) & $8.9( \pm 1.3)$ & $9.1( \pm 1.2)$ & $9( \pm 1.1)$ & 0.84 \\
\hline TAPSE in mm & $22.1( \pm 2.6)$ & $22.9( \pm 2.4)$ & $22.5( \pm 2.5)$ & 0.39 \\
\hline GLS $\%$ & $-19.3( \pm 2.4)$ & $-19.8( \pm 2.3)$ & $-19.4( \pm 2.2)$ & 0.59 \\
\hline
\end{tabular}

ANOVA test.

Regard GLS, the reduction in GLS after 3 week from the first dose by $8 \%-15 \%$ from baseline was found in $6(15 \%)$ patients, while reduction $\geq 15 \%$ from the baseline GLS was found in $2(5 \%)$ patients only figure $-1-$.

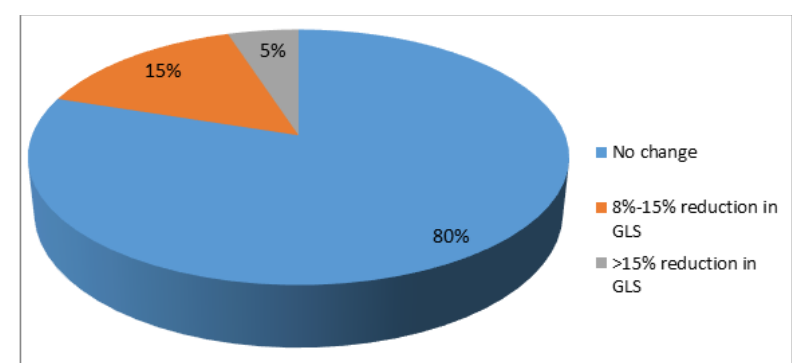

Figure -1- PIE chart for the percentage of reduction in GLS after 3 weeks from the first dose.

The reduction in GLS after 3 week from the second dose by $8 \%-15 \%$ from baseline was found in 2 additional patients, while reduction $\geq 15 \%$ from baseline GLS wasn't found in any additional patients, figure -2-.

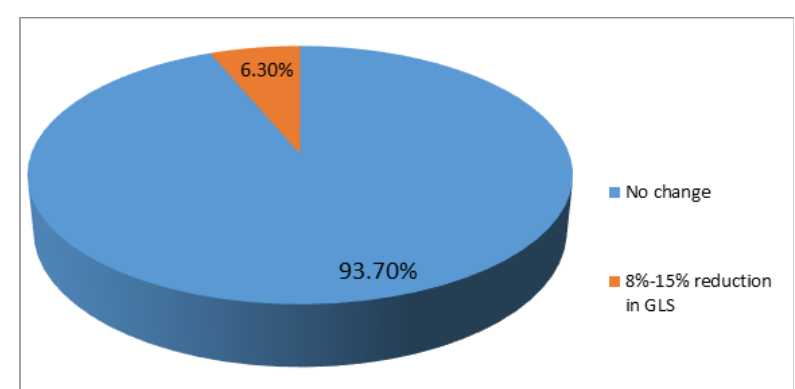

Figure -2- PIE chart for the percentage of decreasing in GLS 3 weeks after the second dose.

\section{Discution}

This study was considered as low morbidity profiles and the risk factors that could be related to cardiotoxicity was relatively low( mean age 51 years, mean BMI was $20.5 \mathrm{Kg} / \mathrm{m}^{2}$, history of DM only in $12.5 \%$, history of dyslipidemia only in $10 \%$, no hypertension, no smoking, no arrhythmia, no previous chemotherapy or radiation). The incidence of cardiotoxicity is fundamental, because it is not uniform in different studies. The cardiotoxicity incidence in a systematic review published in 2014 ranged from $13 \%$ to $32 \%{ }^{(12)}$. Studies published by US at $2011^{(19)}$ and at $2013^{(20)}$ have found an incidence of $20 \%$. Other study found a cardiotoxicity incidence of $10 \%$, lower than that reported by those studies ${ }^{(14)}$. That could be explained by the different morbidity profile of population studied and different age, type of cancer, strain technique and timing of follow up. Despite this hetrogenicity, all uniformly demonstrate that changes in myocardial deformation occur earlier than change in LVEF.

In this study, reduction in the longitudinal strain level was found in $20 \%$ of patients three weeks after the first dose and in $6.3 \%$ of additional patients three weeks after the second dose and without any decrease in systolic function were noted via conventional echocardiography this similar to many other studies like Iranian study that show considerable reduction in GLS in $13 \%$ of patients ${ }^{(21)}$, Ireland study showed, 18 (26\%) of the chemotherapy group, global longitudinal strain was below the lower limit of the control group ${ }^{(22)}$.

Early initiation of heart failure treatment appears important for the recovery of the Left ventricular function in patients who experience a significant reduction in LVEF due to anthracyclines ${ }^{(3)}$. Therefore, early identification of impaired systolic function is vital. 
Furthermore, given that many breast cancer patients first treated with anthracyclines will then receive trastuzumab, identifying patients at greater risk of developing cardiotoxicity is of considerable advantage. Early identification of those with significant reductions in strain would enable targeted monitoring, together with the institution of supportive therapy with angiotensinconverting enzyme-inhibitors or $\beta$-blockers, should further treatment with trastuzumab be required ${ }^{(23)}$.

In this study, there was no significant reduction in global longitudinal strain with preserved LVEF , this compatible to result of Australian study (23) and this contrary to other study which showed considerable reduction in GLS after chemotherapy like Iranian study which found considerable reduction in GLS before and after treatment ${ }^{(24)}$ and European study which also found Global systolic strain was significantly reduced immediately after, and 6 months after anthracyclines (25). the reason behind this may be due to short follow up period 6 week only, we studied patients after anthracycline treatment only (with no radiotherapy or adjuvant trastuzumab) and the patients received only $120 \mathrm{mg} / \mathrm{m}^{2}$ cumulative dose of doxorubicin where the Cardiotoxicity related to anthracycline treatment has strong positive correlation with a cumulative dose.

Conclusion: There was a reduction in GLS between $8 \%-15 \%$ from the baseline in 8 patients and a reduction in GLS between $>15 \%$ from the baseline in 2 patients throughout study period and there were no significant differences in the ejection fraction.

Ethical Clearance: The Research Ethical Committee at scientific research by ethical approval of both environmental and health and higher education and scientific research ministries in Iraq

Conflict of Interest: The authors declare that they have no conflict of interest.

Funding: Self-funding

\section{References}

1. WHO. BREAST CANCER 2019.

2. S Gillespie H, JMcGann C, DWilson B. Noninvasive diagnosis of chemotherapy related cardiotoxicity. Current cardiology reviews. 2011;7(4):234-44.

3. Cardinale D, Colombo A, Lamantia G, Colombo N, Civelli M, De Giacomi G, et al. Anthracyclineinduced cardiomyopathy: clinical relevance and response to pharmacologic therapy. Journal of the American College of Cardiology. 2010;55(3):21320.

4. Mercuro G, Cadeddu C, Piras A, Dessi M, Madeddu C, Deidda M, et al. Early epirubicininduced myocardial dysfunction revealed by serial tissue Doppler echocardiography: correlation with inflammatory and oxidative stress markers. The oncologist. 2007;12(9):1124-33.

5. Albini A, Pennesi G, Donatelli F, Cammarota R, De Flora S, Noonan DM. Cardiotoxicity of anticancer drugs: the need for cardio-oncology and cardiooncological prevention. Journal of the National Cancer Institute. 2010;102(1):14-25.

6. Zamorano JL, Lancellotti P, Rodriguez Muñoz D, Aboyans V, Asteggiano R, Galderisi M, et al. 2016 ESC Position Paper on cancer treatments and cardiovascular toxicity developed under the auspices of the ESC Committee for Practice Guidelines: The Task Force for cancer treatments and cardiovascular toxicity of the European Society of Cardiology (ESC). European heart journal. 2016;37(36):2768-801.

7. Yancy CW, Jessup M, Bozkurt B, Butler J, Casey DE, Drazner MH, et al. 2013 ACCF/AHA guideline for the management of heart failure: a report of the American College of Cardiology Foundation/ American Heart Association Task Force on Practice Guidelines. Journal of the American College of Cardiology. 2013;62(16):e147-e239.

8. Thavendiranathan P, Grant AD, Negishi T, Plana JC, Popović ZB, Marwick TH. Reproducibility of echocardiographic techniques for sequential assessment of left ventricular ejection fraction and volumes: application to patients undergoing cancer chemotherapy. Journal of the American College of Cardiology. 2013;61(1):77-84.

9. Cardinale D, Colombo A, Torrisi R, Sandri MT, Civelli M, Salvatici M, et al. Trastuzumab-induced cardiotoxicity: clinical and prognostic implications of troponin I evaluation. Journal of Clinical Oncology. 2010;28(25):3910-6.

10. Fallah-Rad N, Lytwyn M, Fang T, Kirkpatrick I, Jassal DS. Delayed contrast enhancement cardiac magnetic resonance imaging in trastuzumab induced cardiomyopathy. Journal of Cardiovascular Magnetic Resonance. 2008;10(1):5.

11. FLORESCU M, CINTEZA M, VINEREANU D. 
Chemotherapy-induced cardiotoxicity. Maedica. 2013;8(1):59.

12. Thavendiranathan $\mathrm{P}$, Poulin F, Lim K-D, Plana JC, Woo A, Marwick TH. Use of myocardial strain imaging by echocardiography for the early detection of cardiotoxicity in patients during and after cancer chemotherapy: a systematic review. Journal of the American College of Cardiology. 2014;63(25 Part A):2751-68.

13. Tan TC, Bouras S, Sawaya H, Sebag IA, Cohen $\mathrm{V}$, Picard MH, et al. Time trends of left ventricular ejection fraction and myocardial deformation indices in a cohort of women with breast cancer treated with anthracyclines, taxanes, and trastuzumab. Journal of the American Society of Echocardiography. 2015;28(5):509-14.

14. Gripp EdA, Oliveira GEd, Feijó LA, Garcia MI, Xavier SS, Sousa ASd. Global longitudinal strain accuracy for cardiotoxicity prediction in a cohort of breast cancer patients during anthracycline and/ or trastuzumab treatment. Arquivos brasileiros de cardiologia. 2018;110(2):140-50.

15. Singal P, Li T, Kumar D, Danelisen I, Iliskovic N. Adriamycin-induced heart failure: mechanisms and modulation. Molecular and cellular biochemistry. 2000;207(1-2):77-86.

16. Cochera F, Dinca D, Bordejevic DA, Citu IM, Mavrea AM, Andor M, et al. Nebivolol effect on doxorubicin-induced cardiotoxicity in breast cancer. Cancer management and research. 2018;10:2071.

17. Eschenhagen T, Force T, Ewer MS, De Keulenaer GW, Suter TM, Anker SD, et al. Cardiovascular side effects of cancer therapies: a position statement from the Heart Failure Association of the European Society of Cardiology. European journal of heart failure. 2011;13(1):1-10.

18. Lang RM, Badano LP, Mor-Avi V, Afilalo J, Armstrong A, Ernande L, et al. Recommendations for cardiac chamber quantification by echocardiography in adults: an update from the American Society of Echocardiography and the European Association of Cardiovascular Imaging. European Heart Journal-Cardiovascular Imaging. 2015;16(3):233-71.
19. Sawaya H, Sebag IA, Plana JC, Januzzi JL, Ky $\mathrm{B}$, Cohen V, et al. Early detection and prediction of cardiotoxicity in chemotherapy-treated patients. The American journal of cardiology. 2011;107(9):1375-80.

20. Baratta S, Damiano MA, Marchese ML, Trucco JI, Rizzo MM, Bernok F, et al. Serum markers, conventional Doppler echocardiography and two-dimensional systolic strain in the diagnosis of chemotherapy-induced myocardial toxicity. Argentine Journal of Cardiology. 2013;81(2):1338.

21. Toufan $M$ e. Two- diamentional strain echocardiography for detection of cardiotoxicity in breast cancer patients undergoing chemotherapy. J cardiovascular thorac Res 2017;9(1):29-34.

22. Ho E, Brown A, Barrett P, Morgan RB, King G, Kennedy MJ, et al. Subclinical anthracycline-and trastuzumab-induced cardiotoxicity in the longterm follow-up of asymptomatic breast cancer survivors: a speckle tracking echocardiographic study. Heart. 2010;96(9):701-7.

23. Stoodley PW, Richards DA, Hui R, Boyd A, Harnett PR, Meikle SR, et al. Two-dimensional myocardial strain imaging detects changes in left ventricular systolic function immediately after anthracycline chemotherapy. European journal of echocardiography. 2011;12(12):945-52.

24. Mirszaee F ea. speckle tracking echocardiography for detection of early myocardial changes in patients treated with anthracyclines INT CARDIOVASCULAR RESJ 2014 11(2):55-9.

25. Stoodley PW, Richards DA, Boyd A, Hui R, Harnett PR, Meikle SR, et al. Left ventricular systolic function in HER2/neu negative breast cancer patients treated with anthracycline chemotherapy: a comparative analysis of left ventricular ejection fraction and myocardial strain imaging over 12 months. European journal of cancer. 2013;49(16):3396-403. 